\title{
Adaptabilidade e estabilidade de progênies de meios-irmãos de pinhão-manso em diferentes regiões do Brasil
}

\author{
Rodrigo Barros Rocha ${ }^{1 *}$, Bruno Galvêas Laviola ${ }^{2}$, Sergio Delmar dos Anjos e Silva ${ }^{3}$, Ana Cristina Pinto Juház, \\ Júlio César Albrecht ${ }^{5}$, Tatiana Barbosa Rosado ${ }^{6}$
}

$10.1590 / 0034-737 X 201663020008$

\begin{abstract}
RESUMO
Programas de melhoramento do pinhão-manso (Jatropha curcas L.) intensificaram-se nos últimos cinco anos, tendo sido selecionadas, localmente, plantas em diversas regiões do Brasil. O objetivo deste trabalho foi quantificar a interação genótipos $\mathrm{x}$ ambientes da produção de grãos de pinhão-manso, avaliada em três regiões brasileiras, e o progresso genético obtido com a seleção. A partir de progênies de meios-irmãos, selecionadas pela Embrapa Semiárido e pela EPAMIG, foram instalados, no ano de 2008, três testes de progênies, nos municípios de Planaltina, DF, Nova Porteirinha, MG e Pelotas, RS, utilizando-se delineamento de blocos ao acaso, com três repetições e cinco plantas por parcela. Como testemunhas foram utilizadas sementes de plantas não selecionadas e um dos materiais genéticos comercializados no Brasil. A interação genótipo $\mathrm{x}$ ambiente foi significativa. Foram identificadas oito progênies de adaptabilidade geral, três progênies de baixa adaptabilidade, duas progênies de adaptabilidade específica a ambientes favoráveis e duas progênies de adaptabilidade específica a ambientes desfavoráveis, em diferentes regiões do Brasil. As estimativas de progresso genético indicam eficiência da seleção massal, com ganhos de 28, 76 e 177\%, nos municípios de Planaltina, DF, de Nova Porteirinha, MG, e de Pelota, RS, respectivamente. Observa-se que os ganhos de seleção obtidos pelo método centroide são mais equilibrados entre ambientes e, por isso, preferíveis. As novas médias, estimadas com o plantio das progênies selecionadas, em toneladas por hectare, são de 2,34 ton.ha ${ }^{-1}$, em Planaltina, DF; de 2,37 ton.ha ${ }^{-1}$, em Nova Porteirinha, MG, e de 2,09 ton.ha-1 ${ }^{-1}$ em Pelotas, RS.
\end{abstract}

Palavras-chave: Jatropha curcas L., interação genótipo ambiente, progresso genético.

\begin{abstract}
Adaptability and stability of Jatropha curcas L. progenies in different regions of Brazil

Physic nut (Jatropha curcas L.) breeding programs have intensified in the past five years, locally selecting plants from various Brazilian regions. The objective of this study was to quantify the genotype $\mathrm{x}$ environment interaction of the physic nut grain production and the genetic progress obtained with the selection. From Half-sib progenies selected by Embrapa and EPAMIG, in 2008, three progeny trials were installed in the cities of Planaltina-DF, Nova PorteirinhaMG and Pelotas-RS, using a randomized block design with three replications of five plants per plot. Non-selected plant seeds and genetic material commercialized in Brazil were used as control. The genotype $\mathrm{x}$ environment interaction was significant for the J. curcas grain yield expression. We identified eight progenies of broad adaptability, three progenies of low adaptability, two progenies of specific adaptability to favorable environments and two progenies of specific
\end{abstract}

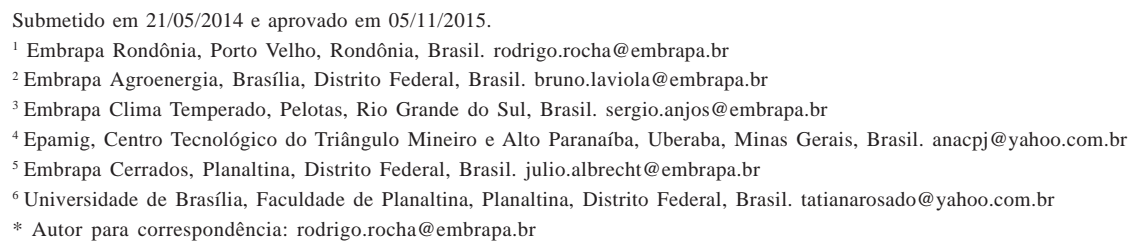


adaptability to unfavorable environments of different Brazilian regions. Estimates of genetic progress indicate mass selection efficiency, with genetic gains of 28\%, $76 \%$ and $177 \%$ in the Planaltina-DF, New Porteirinha-MG and PelotasRS, respectively. The genetic gains obtained by the centroid method were more balanced among environments, and therefore, preferable. The new means estimated with the cultivating of the selected progenies are: 2.34 ton.ha $^{-1}$ in Planaltina-DF, 2.37 ton.ha ${ }^{-1}$ in Nova Porteirinha- MG and 2.09 ton.ha" ${ }^{-1}$ in Pelotas-RS.

Key words: Jatropha curcas L., genotype environment interaction, genetic progress.

\section{INTRODUÇÃO}

A resposta diferencial das plantas às variações ambientais faz com que estudos da interação genótipos $\mathrm{x}$ ambientes sejam necessários para os programas de melhoramento de diversas culturas. Efeitos significativos da interação genótipos x ambientes são resultado da relação não aditiva dos efeitos de genótipo e de ambiente, causada pela mudança do desempenho das plantas em diferentes locais (Resende, 2002). Segundo Cruz et al. (2004), programas de melhoramento de plantas devem procurar desenvolver cultivares que apresentam ampla adaptabilidade e boa estabilidade, sendo o termo adaptabilidade definido como a capacidade dos genótipos de responder à melhoria do ambiente e o termo estabilidade definido como a previsibilidade do comportamento em diferentes ambientes.

Considerado como uma espécie de interesse para produção de óleo vegetal, o pinhão-manso é uma oleaginosa perene com óleo de excelente qualidade. Segundo Spinelli et al. (2010), a produtividade de grãos dessa oleaginosa é a característica mais importante para a seleção de plantas de maior rendimento de óleo, seguida, em menor importância, pelo volume de copa e pelo teor de óleo dos grãos. Da expectativa inicial, de quatro ou mais toneladas de grãos por hectare, produtividades inferiores a duas toneladas por hectare estão sendo obtidas, em diferentes condições edafoclimáticas, por causa das limitações hídricas, dos ataques de pragas e doenças e do cultivo de plantas não selecionadas (Laviola \& Dias, 2008; Drumond et al., 2010; Gouveia et al., 2012; Filho et al., 2013).

Programas de melhoramento do pinhão-manso, no Brasil e no mundo, ainda estão em fase inicial e pouco se conhece sobre a interação genótipos $\mathrm{x}$ ambientes. A incidência de oídio em regiões de cerrado e o ataque da cigarrinha têm sido observados em plantios em diferentes regiões do Brasil (Juhász et al., 2010; Freitas et al., 2011; Borges et al., 2014). Com o objetivo de selecionar materiais superiores, a Embrapa e a rede de pesquisa do projeto Pesquisa, Desenvolvimento e Inovação em Pinhão-Manso para a produção de Biodiesel (BRJATROPHA/FINEP/ $\mathrm{MCTI} / \mathrm{CNPq}$ ) vem realizando ensaios em diferentes ambi- entes, com objetivo de selecionar plantas adaptadas a diferentes regiões do Brasil (Laviola et al., 2012).

Pesquisado em todo o mundo como uma espécie de interesse para produção de óleo (Divakara et al., 2010; Carels et al., 2013), programas de melhoramento de pinhãomanso intensificaram-se nos últimos cinco anos, no Brasil, (Juhász, et al., 2013). Nesse período, progênies de meios-irmãos (FMI) foram selecionadas por seleção massal, na Embrapa Semiárido (CPATSA) (Drumond et al., 2010), em Petrolina, PE, e na EPAMIG, em Nova Porteirinha, MG, (Juhász et al., 2010). A seleção massal positiva fundamenta-se na identificação de indivíduos com o fenótipo desejado para formar uma nova população.

Apesar da maior facilidade de condução, a seleção massal apresenta menor acurácia para características de baixa herdabilidade e de interação alélica não aditiva (Resende et al., 2001; Resende, 2007). A eficiência da seleção massal pode ser estimada pela diferença do desempenho da população selecionada em comparação com o da população original.

Existem disponíveis na literatura vários métodos para quantificação da interação genótipos $\mathrm{x}$ ambientes, entre os quais se destacam os métodos baseados na análise da variância (Plaisted \& Peterson, 1959), na regressão linear simples (Finlay \& Wilkinson, 1963; Eberhart \& Russel, 1966), na regressão linear múltipla (Verma et al., 1978; Cruz et al., 1989), nos métodos não-paramétricos (Lin \& Binns, 1988) e nos multivariados (Rocha et al., 2005; Nascimento et al., 2009).

Cruz \& Castoldi (1991) apresentaram uma nova decomposição do quadrado médio da interação genótipos x ambientes, em suas partes simples e complexa. A interação genótipos $\mathrm{x}$ ambientes de natureza simples é resultado da variabilidade genética entre as progênies em avaliação e que não resulta em mudanças no ordenamento das progênies mais produtivas de um ambiente para o outro. Já a interação de natureza complexa é causada pelo desempenho não previsível nos diferentes ambientes, que resulta em uma inconsistência da superioridade das progênies nos diferentes ambientes (Cruz et al., 2004, Falconer, 1987). A quantificação das frações simples e complexa da interação genótipos $\mathrm{x}$ ambientes permite agrupar os ambientes de 
interação não significativa (Lin, 1982). A dispersão no plano do método centroide permite comparar o desempenho das progênies com pontos de referência de máxima adaptabilidade e estabilidade, auxiliando o melhorista a identificar genótipos de adaptabilidade geral ou específica.

Diante do exposto, o objetivo deste trabalho foi quantificar a interação genótipos $\mathrm{x}$ ambientes da produção de grãos de progênies de pinhão-manso, avaliadas em três diferentes regiões brasileiras, e o progresso genético obtido com a seleção.

\section{MATERIAL E MÉTODOS}

\section{Experimentação agrícola}

Em fevereiro de 2008, foram instalados três testes de progênies para a avaliação do desempenho de 18 progênies de meios-irmãos (FMIs), nos municípios de Planaltina, DF, Nova Porteirinha, MG e Pelotas, RS, utilizando-se delineamento em blocos ao acaso, com três repetições de cinco plantas por parcela, dispostas em linha. O espaçamento adotado foi de quatro metros entre linhas e de dois metros dentro da linha de plantio. Todas as práticas de manejo foram baseadas em Dias et al. (2007), com adaptações, de acordo com os recentes avanços da pesquisa com pinhão-manso no Brasil (Bahadur et al., 2013; Carels et al., 2013; Resende et al., 2013).

Como testemunhas, foram utilizados o tratamento 1 (CNPAE-154), que representa o primeiro material genético comercializado, no Brasil, pela Empresa Biojan de Janaúba, MG (Tominaga et al., 2007) e o tratamento 2 (CPATSA-C C $_{0}$, obtido a partir de uma mistura de sementes sem seleção.

Os tratamentos identificados com as numerações de 3 a 18 representam progênies selecionadas para maior produção de grãos, por seleção massal, pela Embrapa Semiárido (CPATSA), no município de Petrolina, PE, e pela EPAMIG, no município de Nova Porteirinha, MG (Tabela 1).

Para avaliação do desempenho agronômico, foi considerada a produção de grãos do ano agrícola de 2012/2013, que corresponde ao terceiro ano de produção das progênies de pinhão-manso. As parcelas foram avaliadas para produção de grãos (g.planta ${ }^{-1}$, posteriormente convertidas em ton.ha ${ }^{-1}$ ). Para isso, foram realizadas de duas a quatro colheitas de frutos maduros, uma vez que o número de colheitas variou com a região de avaliação.

\section{Análise de variância}

A significância dos efeitos de progênies, testemunhas e ambientes e da interação progênies $\mathrm{x}$ ambientes da produção de grãos foram avaliados, considerando-se o efeito de progênies como aleatório e o efeito de ambientes como fixo, utilizando-se, nas análises individuais, o modelo $Y_{i j}=$ $m+T_{i}+B_{k}+\varepsilon i_{i k}$,em que: $Y_{i j k}$ : produção da i-ésima parcela no K-ésimo bloco, $m$ : média geral, $B_{k}$ : efeito do k-ésimo bloco, $G_{i}$ : efeito do i-ésimo tratamento (progênie ou testemunha), $\varepsilon_{i k}$ : erro aleatório associado a cada observação ik (Cruz et al., 2004).

$\mathrm{Na}$ análise conjunta, foi utilizado o modelo $Y_{i j k}=m+$ $(B / A)_{j k}+T_{i}+A_{j}+G A_{i j}+\varepsilon i_{j k}$, em que: $Y_{i j k}$ : produção da iésima parcela no k-ésimo bloco e no j-ésimo ambiente, $m$ : média geral, $(B / A)_{j k}$ : efeito do k-ésimo bloco no j-ésimo ambiente, $G_{i}$ : efeito do i-ésimo tratamento (progênie ou testemunha), $A_{j}$ : efeito do j-ésimo ambiente, $G A_{i j}$ : efeito da interação entre a i-ésima progênie e o j-ésimo ambiente, $\varepsilon i_{j k}$ : erro aleatório associado a observação ijk. A homogeneidade das variâncias foi verificada pelo teste de Bartlett (Cruz et al., 2004).

\section{Estratificação ambiental}

A análise de estratificação ambiental foi realizada pelos métodos de Lin (1982), para agrupamento dos ambientes, e de Cruz \& Castoldi (1991), para quantificar as frações simples e complexa da interação genótipos x ambientes. Lin (1982) propôs a utilização de um estimador, com a mesma distribuição do teste F, para testar a hipótese de igualdade entre soma de quadrados da interação genótipos $\mathrm{x}$ ambientes de diferentes locais, agrupando os ambientes de interação não significativa:

$$
F_{j j^{\prime}}=\frac{\left[S Q G A_{i j j^{\prime}} /(n-1)(g-1)\right]}{(Q M R / r)}
$$

em que: : soma de quadrados entre genótipos (progênies) e pares de ambientes, número de observações, : número de genótipos (progênies), quadrado médio do resíduo, número de repetições.

Tabela 1: Identificação das progênies de meios-irmãos avaliadas nos municípios de Planaltina, DF, Nova Porteirinha, MG, e Pelotas, RS

\begin{tabular}{lc}
\hline Tratamento & Identificação \\
\hline 1 & CNPAE-154 $($ Controle) \\
2 & CPATSA-C $_{0}($ Controle) \\
3 & CPATSA-C $_{1}($ P1-L3) \\
4 & CPATSA-C $_{1}(\mathrm{P} 3-L 4)$ \\
5 & CPATSA-C $_{1}(\mathrm{P} 2-\mathrm{L} 3)$ \\
6 & CPATSA-C $_{1}(\mathrm{P} 12-\mathrm{L} 6)$ \\
7 & CPATSA-C $_{1}(\mathrm{P} 13-\mathrm{L} 4)$ \\
8 & CPATSA-C $_{1}(\mathrm{P} 6-\mathrm{L} 7)$ \\
9 & CPATSA-C $_{1}(\mathrm{P} 4 / \mathrm{L} 3)$ \\
10 & CPATSA-C $_{1}(\mathrm{P} 10-\mathrm{L} 4)$ \\
11 & CPATSA-C $_{1}(\mathrm{P} 14-\mathrm{L} 5)$ \\
12 & CPATSA-C $_{1}(\mathrm{P} 25 /$ L6) \\
13 & EPAMIG-C $_{1}\left(10 / 3^{\mathrm{a}}\right)$ \\
14 & EPAMIG-C $_{1}(24)$ \\
15 & EPAMIG-C $_{1}\left(34 / 2^{\mathrm{a}}\right)$ \\
16 & EPAMIG-C $_{1}(37)$ \\
17 & EPAMIG-C $_{1}\left(38 / 2^{\mathrm{a}}\right)$ \\
18 & EPAMIG-C $_{1}(54)$ \\
\hline
\end{tabular}


Por sua vez, Cruz \& Castoldi (1991) apresentaram uma nova decomposição do quadrado médio da interação em suas partes simples e complexa obtida, pela expressão, em que r é a correlação entre o desempenho médio dos genótipos nos locais 1 e 2, e, são os quadrados médios dos genótipos nos locais 1 e 2 respectivamente. A interação de natureza simples é resultado da variabilidade genética entre os genótipos em avaliação, enquanto a interação de natureza complexa é causada pelo desempenho não previsível dos genótipos nos diferentes ambientes, que resulta em uma inconsistência na superioridade dos genótipos nos diferentes ambientes (Cruz et al., 2004). Visando a identificar os ambientes favoráveis e desfavoráveis ao cultivo, foi utilizado o índice ambiental proposto por Finlay e Wilkinson (1963):

$$
I_{j}=\frac{1}{g} \sum_{i} Y_{i j}-\frac{1}{a g} Y,
$$

em que: :média da i-ésima progênie, no j-ésimo ambiente; : total das observações; = número de ambientes; = número de genótipos.

\section{Seleção de plantas e quantificação do progresso com a seleção}

Método centroide (Rocha et al., 2005; Nascimento et al., 2009): método que utiliza técnica multivariada para obter um número reduzido de variáveis abstratas e independentes, que permite comparar no plano o desempenho de todas as progênies em relação a quatro referências ideais (ideótipos). A referência de máxima adaptabilidade geral é aquela que apresenta valores máximos observados em todos os ambientes (ideótipo I). As referências de máxima adaptabilidade específica são aquelas que: apresentam máximo desempenho em ambientes favoráveis e mínimo desempenho em ambientes desfavoráveis (ideótipo II); mínimo desempenho em ambientes favoráveis e máxima resposta em ambientes desfavoráveis (ideótipo III). A referência de mínima adaptabilidade é aquela que apresenta os menores valores observados em todos os ambientes (ideótipo IV). As progênies foram classificadas de acordo com sua proximidade a cada um dos centroides (Rocha $e t$ al., 2005).

$$
P_{d(i, k)}=\frac{\left(\frac{1}{d_{i k}}\right)}{\sum_{i} \frac{1}{d_{i k}}},
$$

em que: : probabilidade de apresentar padrão de estabilidade semelhante ao k-ésimo centroide e : distância do iésimo genótipo ao k-ésimo centroide, no plano gerado a partir da análise de componentes principais.

\section{Ganho de seleção}

Com o objetivo de comparar o progresso genético com o plantio das progênies de maior adaptabilidade e estabilidade, o progresso genético foi estimado por comparação com a média do plantio, a média da testemunha e a média da população de controle, em que não houve seleção. A resposta indireta à seleção, em cada ambiente, foi estimada de acordo com (Cruz, 2004; Resende, 2007):

$G S_{j(i)}=D S_{j(i)} h_{2 j}$,

em que: : ganho de seleção indireto no ambiente $\mathrm{j}$ resultado da seleção direta no ambiente i, : diferencial de seleção indireto obtido a partir do contraste entre a média da população e dos indivíduos selecionados no ambiente i; : herdabilidade no ambiente $\mathrm{j}$.

\section{RESULTADOS E DISCUSSÃO}

O estudo dos padrões de herança de características de produção indica que, para se obter maior eficiência de seleção, deve-se primeiro selecionar plantas de maior potencial produtivo, para somente então, avaliar a adaptabilidade e estabilidade em um número maior de ambientes (Cruz, 2004). As análises de variância individuais mostram que o efeito de progênies foi significativo em todos os ambientes, indicando diferença no seu potencial produtivo (Tabela 2). A significância do efeito testemunha, nos ambientes de Planaltina, DF e de Nova Porteirinha, MG, indica que as testemunhas apresentaram comportamento diferenciado e que a significância do contraste progênies $\mathrm{x}$ testemunha deve ser interpretado separadamente para cada uma das testemunhas utilizadas (Tabela 2).

A testemunha CNPAE-154 apresentou média superior à da testemunha CPATSA-C0, em todos os ambientes (Tabela 2). Tendo em vista que a primeira testemunha é um material comercializado e, a segunda, uma mistura de sementes não selecionadas, o ganho com a seleção das progênies deve ser interpretado em comparação com as duas testemunhas, para se quantificar a eficiência da seleção e o ganho esperado.

O resultado do teste de Bartlett não foi significativo, indicando a homogeneidade das variâncias nos ambientes avaliados (Tabela 2). A análise conjunta indicou a significância dos efeitos de progênies, de testemunhas e de ambientes e da interação progênies x ambientes. A ocorrência de efeitos significativos da interação progênies $x$ ambientes indica comportamento inconsistente e diferenciado das progênies, nas regiões estudadas. Apenas a interação testemunhas $\mathrm{x}$ ambientes não foi significativa, indicando consistência na superioridade da primeira testemunha (CNPAE-154), em comparação com a segunda (CPATSA-C0), em todos os ambientes. 
A interação genótipos x ambientes é importante fator da diferença da variância genética entre ambientes e da baixa correlação entre os tratamentos genéticos de um ambiente para outro. Segundo Resende (2007), a interação do tipo simples não acarreta problemas para a seleção, enquanto a do tipo complexo limita a seleção de plantas de adaptação geral.

A decomposição das frações simples e complexas da interação genótipos $\mathrm{x}$ ambientes indicou que a maior parte da interação complexa está associada ao município de Pelotas, RS (Tabela 3). Em termos de desempenho, verifica-se que a média geral do experimento também foi menor no município de Pelotas, RS, comparada com a dos ensaios realizados em Planaltina, DF e em Nova Porteirinha, MG. Este resultado é esperado, já que o pinhão-manso é uma planta de clima tropical e de menor adaptação às temperaturas da região sul do Brasil (Tabela 3). Também considera-se que as progênies foram selecionadas pela Embrapa Semiárido e pela EPAMIG, em ambientes que apresentam temperaturas médias superiores a $23{ }^{\circ} \mathrm{C}$. A análise de estratificação pelo método de Lin (1982) indicou a formação de dois grupos, um primeiro, formado por Planaltina, DF e Nova Porteirinha, MG, que apresentou a maior parte da interação genótipos $\mathrm{x}$ ambientes de natureza simples, e um segundo grupo, formado apenas pelo ambiente de Pelotas, RS.

Os ambientes em que foram instalados os ensaios, nos municípios de Pelotas, RS, Planaltina, DF e Nova Porteirinha, MG, apresentam naturalmente grandes diferenças edafoclimáticas (Tabela 4). O índice ambiental clas-

Tabela 2: Resumo das análises de variância para os ambientes de Planaltina, DF, Nova Porteirinha, MG, e Pelotas, RS, da produção de grãos (g.planta-1)

\begin{tabular}{lcccc}
\hline & \multicolumn{3}{c}{ Análises individuais } & \\
FV & $\mathbf{G L}$ & $\mathbf{F}_{\mathbf{1}}$ & $\mathbf{F}_{\mathbf{2}}$ & $\mathbf{F}_{\mathbf{3}}$ \\
\hline BLOCOS & 2 & - & - & - \\
TRATAMENTOS (T) & 17 & $10,76^{* *}$ & $6,62^{* *}$ & $2,64^{* *}$ \\
$\quad$ Progênies (P) & 15 & $11,82^{* *}$ & $6,89^{* *}$ & $2,18^{* *}$ \\
$\quad$ Testemunha (T) & 1 & $4,32^{* *}$ & $8,23^{* *}$ & $1,20^{\mathrm{NS}}$ \\
Prog. x Test. (PxT) & 1 & $1,21^{\mathrm{NS}}$ & $0,92^{\mathrm{NS}}$ & $-11,07^{* *}$ \\
RESÍDUO & 34 & - & - & 1302,21 \\
\hline Média Geral & & 1543,78 & 1536,23 & 1374,95 \\
Média das Progênies & & 1531,91 & 1764,56 & 923,96 \\
Média CNPAE-154 (Controle) & & 1828,60 & 1056,40 & 516,74 \\
Média CPATSA-C & (Controle) & 1448,76 & 19,84 & 34,88 \\
CVe (\%) & & 14,48 & &
\end{tabular}

\begin{tabular}{lcc}
\hline & Análise conjunta & \\
\hline FV & GL & $\mathbf{F}_{\mathbf{c}}$ \\
BLOCOS/AMB & 6 & - \\
TRATAMENTOS (T) & 17 & $10,83^{* *}$ \\
$\quad 15$ & $11,16^{* *}$ \\
$\quad$ Progênies (P) & 1 & $9,64^{* *}$ \\
$\quad$ Testemunha (T) & 1 & $6,96^{* *}$ \\
$\quad$ Grupos (G) & 2 & $1,84^{\mathrm{NS}}$ \\
AMBIENTES (A) & 34 & $1,87^{* *}$ \\
TRAT x AMBIENTES & 30 & $1,62^{*}$ \\
Prog. xAmb. (PxA) & 2 & $0,42^{\mathrm{NS}}$ \\
Test. x Amb. (TxA) & 2 & $7,01^{* *}$ \\
$\quad$ Grupos x Amb. (GxA) & 102 & 1456,08 \\
RESÍDUO & & 1481,04 \\
\hline Média Geral & & 1505,70 \\
Média das Progênies & & 1007,29 \\
Média CNPAE-154 (Controle) & & $0,82^{\mathrm{NS}}$ \\
Média CPATSA-C $($ Controle) & & 23,37 \\
Teste de Bartlett & & \\
CVe (\%) & & \\
\hline
\end{tabular}

FV: fonte de variação, GL: graus de liberdade, F: teste F, **: significativo a $1 \%$ de probabilidade de acordo com o teste $\mathrm{F}$, *: significativo a $5 \%$ de probabilidade de acordo com o teste $\mathrm{F}$, Ns: não significativo, $\mathbf{F}_{\mathbf{1}}$ :Estimativa do teste $\mathrm{F}$ para o ambiente de Planaltina - DF, $\mathbf{F}_{2}$ : Estimativa do teste $\mathrm{F}$ para o ambiente de Nova Porteirinha-MG, $\mathbf{F}_{3}$ : Estimativa do teste F para o ambiente de Pelotas-RS, $\mathbf{F}_{\mathrm{c}}$ : Estimativa do teste $\mathrm{F}$ da análise conjunta nos três ambientes, $\mathbf{C V e}(\%)$ : coeficiente de variação experimental. 
sificou os ambientes de Planaltina, DF e Nova Porteirinha, MG, como favoráveis para o cultivo do pinhão-manso em comparação com o ambiente de Pelotas, RS. Os ensaios conduzidos nos ambientes de Planaltina e de Nova Porteirinha proporcionaram menores coeficientes de variação experimental e maior herdabilidade do caráter produção de grãos, em comparação com os do ensaio conduzido em Pelotas, que apresenta condições menos favoráveis para a seleção (Tabela 4).

A identificação de progênies responsivas à melhoria do ambiente, que mantém sua superioridade em diferentes regiões, subsidia recomendações mais amplas de plantio. Como estas plantas nem sempre podem ser encontradas nas populações de melhoramento, procura-se discriminar as progênies de adaptabilidade geral e específica daquelas de baixa adaptabilidade. Com a caracterização dos efeitos de progênies, de ambientes e da interação progênies $x$ ambientes, o método centroide foi utilizado para selecionar as progênies de maior adaptabilidade e estabilidade. Este método utiliza a técnica de componentes principais para a obtenção de um número reduzido de variáveis abs- tratas e independentes, visando a representar em ordem de estimação o máximo da variação total contida nas variáveis originais.

O uso de diferentes métodos para estudo da interação progênies $\mathrm{x}$ ambientes proporciona resultados complementares, que, por este motivo, são interpretados em conjunto. Os conceitos de adaptabilidade e de estabilidade do método centroide fundamentam-se na comparação do desempenho das progênies com pontos de referência, estimados a partir dos próprios dados experimentais (Figura 1). O percentual de variância acumulada pelos dois primeiros componentes principais foi de $94,36 \%$, valor este considerado adequado para interpretação dos dados. As progênies foram classificadas de acordo com sua proximidade a cada um dos centroides, tendo sido encontradas oito progênies de adaptabilidade geral (4, 11, 10, 5, 7, 9, 12 e 3), duas progênies de adaptabilidade específica a ambientes favoráveis $(8,6)$, duas progênies de adaptabilidade específica a ambientes desfavoráveis (14 e 13) e três progênies de baixa adaptabilidade (15, 16 e 17). Especificamente, observa-se que o CNPAE-154, que representa o primeiro ma-

Tabela 3: Estimativas dos percentuais da parte simples e da parte complexa da interação genótipo x ambiente e estratificação ambiental

\begin{tabular}{lcc}
\hline \multicolumn{2}{c}{ Percentuais da parte simples e complexa da interação (Cruz \&Castoldi, 1991) } \\
\hline Ambientes & Simples & Complexa \\
\hline 1.Planaltina, DF x 2.Nova Porteirinha, MG & 66,4 & 33,6 \\
1.Planaltina, DF x 3.Pelotas, RS & 26,1 & 73,9 \\
2.Nova Porteirinha, MG x 3.Pelotas, RS & 29,6 & 70,4 \\
\hline
\end{tabular}

\begin{tabular}{lcc}
\hline & Estratificação ambiental (Lin, 1982) & \\
\hline Ambientes & Grupos & Fcalc \\
\hline 1.Planaltina, DF, 2.Nova Porteirinha, MG & $\mathrm{I}$ & $\mathrm{F}_{12}=1,02^{\mathrm{NS}}$ \\
3.Pelotas, RS & II & $\mathrm{F}_{123}=5,01^{* *}$ \\
\hline
\end{tabular}

$\mathrm{F}_{12}=$ Estimativa do teste $\mathrm{F}$ modificado por Lin, 1982 para testar a hipótese de ocorrência de interação genótipo x ambiente não significativa entre os ambientes de1.Planaltina-DF e 2.Nova Porteirinha-MG, $\mathrm{F}_{123}=$ Estimativa do teste $\mathrm{F}$ modificado por Lin, 1982 para testar a hipótese de ocorrência de interação genótipo x ambiente não significativa entre os ambientes 1.Planaltina-DF, 2.Nova Porteirinha-MG e 3. Pelotas-RS.

Tabela 4: Características edafoclimáticas e parâmetros genéticos estimados em testes de progênies de pinhão-manso instalados nos municípios de Planaltina, DF, Nova Porteirinha, MG, e Pelotas, RS

\begin{tabular}{|c|c|c|c|c|c|}
\hline \multicolumn{6}{|c|}{ Características edafoclimáticas } \\
\hline Ambiente & Clima* & $\begin{array}{l}\mathrm{Tm} \\
\left({ }^{\circ} \mathrm{C}\right)\end{array}$ & $\begin{array}{l}\text { Urm } \\
(\%)\end{array}$ & $\begin{array}{l}\text { Precipitação } \\
\text { média (mm) }\end{array}$ & $\begin{array}{c}\text { Altitude } \\
\text { (m) }\end{array}$ \\
\hline Planaltina-DF & Aw & 21 & 68 & 1.100 & 1.007 \\
\hline Nova Porteirinha-MG & Aw & 23 & 52 & 650 & 518 \\
\hline Pelotas-RS & Cfa & 18 & 80 & 1.100 & 60 \\
\hline \multicolumn{6}{|c|}{ Parâmetros genéticos } \\
\hline Ambiente & $\mathrm{CVg}_{(\%)}$ & $\mathrm{CVr}_{(\%)}$ & $\mathbf{I}_{\mathbf{j}}$ & $\mathbf{S}_{g}^{2}$ & $\mathbf{h}^{2}$ \\
\hline Planaltina-DF & 27,73 & 1,89 & 87,70 & 162737,30 & 0,91 \\
\hline Nova Porteirinha-MG & 27,56 & 1,40 & 66,17 & 171080,60 & 0,85 \\
\hline Pelotas-RS & 20,72 & 0,63 & $-153,87$ & 113213,81 & 0,62 \\
\hline
\end{tabular}

* Classificação climática de Kopen-Geiger, Aw: savana com inverno seco, Cfa: clima subtropical úmido, Tm: temperatura média, Urm: umidade relativa média do $\operatorname{ar}, \mathbf{C V}_{(\%)}$ : coeficiente de variação, $\mathbf{I j}$ : índice ambiental, $\mathrm{S}_{\mathrm{g}}^{2}$ : variância genotípica, $\mathbf{h}^{2}$ : herdabilidade. 
terial genético comercializado no Brasil, apresentou adaptação específica aos ambientes favoráveis (Planaltina, DF e Nova Porteirinha, MG) e que a mistura de sementes de plantas não selecionadas $\left(\mathrm{CPATSA}-\mathrm{C}_{0}\right)$ não respondeu à melhoria das condições de ambiente, apresentando uma média baixa em todos os ambientes, maior apenas que o desempenho da progênie 18 (Figura 1).

As progênies 4, 11, 10, 3 e 12 foram selecionadas, considerando-se a maior proximidade ao ideótipo I. De maneira geral, observa-se superioridade das progênies da Embrapa, selecionadas em Petrolina, PE, que apresentaram maiores adaptabilidade e estabilidade (Figura 1).

O ganho de seleção com o plantio das progênies selecionadas foi interpretado para quantificar a eficiência da seleção entre progênies, em relação à média do experimento e às testemunhas avaliadas (Tabela 4). As estimativas do progresso genético mostram que os ganhos obtidos com a seleção baseada na média e no método centroide são de magnitudes semelhantes. Isto se deve à maior correlação no desempenho das progênies, observada entre os ambientes de Planaltina, DF, e de Nova Porteirinha, MG. No entanto, observa-se que os ganhos de seleção obtidos com as progênies selecionadas pelo método centroide são mais equilibrados entre ambientes, e por isso, preferíveis (Tabela 4).

As estimativas do ganho em relação à população não melhorada (CPATSA-C $\mathrm{C}_{0}$ ) indica eficiência da seleção de progênies realizada nos municípios de Petrolina e Nova Porteirinha, que resultaram em estimativas de ganhos de 28 até $177 \%$. Apesar da baixa diversidade genética quantificada por técnicas de marcadores moleculares (Rosado et al., 2010), avaliações agronômicas têm mostrado variabilidade genética para os principais componentes da produção dessa oleaginosa (Mishra et al., 2009). Ganhos de seleção superiores a $80 \%$ foram estimados por Laviola et al. (2012) e Drumond et al. (2010) na seleção de plantas em um único ambiente.

Menores estimativas de progresso genético foram observadas em comparação com a testemunha CNPAE-154 (Tabela 5). Mesmo considerando-se as baixas produtividades obtidas nos últimos anos com o cultivo do pinhão-

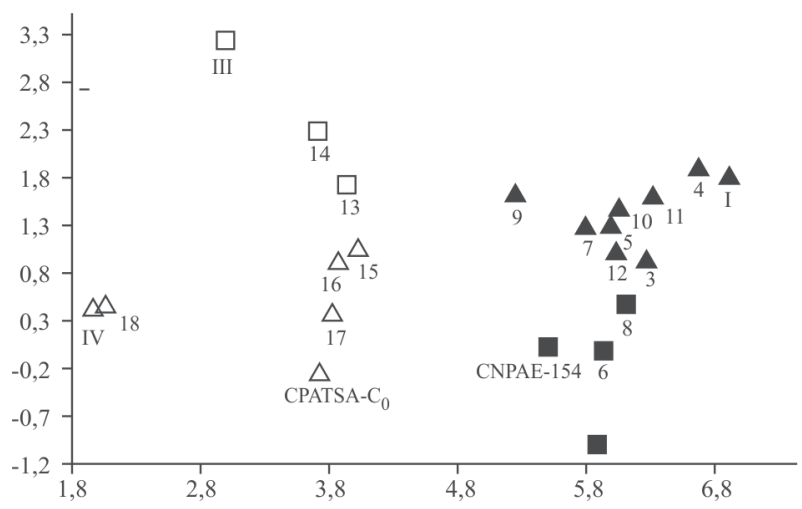

Figura 1: Dispersão gráfica dos dois primeiros componentes principais da produção de grãos de progênies de meios-irmãos de pinhão-manso e de quatro pontos de referência, que indicam os ideótipos de máxima adaptabilidade geral (I), máxima adaptabilidade específica a ambientes favoráveis (II), máxima adaptabilidade específica a ambientes desfavoráveis (III) e baixa adaptabilidade (IV).

Tabela 5: Progresso genético da produção de grãos $\left(\mathrm{g} \cdot\right.$ planta $\left.^{-1}\right)$ com a seleção das melhores progênies em cada ambiente em relação à média do experimento, a média da população não selecionada e em relação à testemunha

\begin{tabular}{|c|c|c|c|c|c|c|}
\hline \multicolumn{7}{|c|}{ Ganho estimado em relação à média } \\
\hline \multirow{2}{*}{ Ambiente } & \multicolumn{6}{|c|}{ Seleção baseada na médiaSeleção baseada no centroide } \\
\hline & $\mathbf{h}_{\mathbf{c}}$ & GS & $\mathbf{G S}_{\%}$ & $\mathbf{h}_{\mathbf{c}}$ & GS & $\mathbf{G S}_{\%}$ \\
\hline Planaltina(DF) & 0,95 & 241,5 & 15,6 & 0,95 & 313,0 & 20,3 \\
\hline Nova Porteirinha(MG) & 0,92 & 396,7 & 26,1 & 0,92 & 372,0 & 24,1 \\
\hline Pelotas(RS) & 0,79 & 310,4 & 23,8 & 0,79 & 294,1 & 22,6 \\
\hline \multicolumn{7}{|c|}{ Ganho estimado em relação à população $\mathrm{C}_{0}($ CPATSA-C $)$} \\
\hline \multirow{2}{*}{ Ambiente } & \multicolumn{6}{|c|}{ Seleção baseada na médiaSeleção baseada no centroide } \\
\hline & $\mathbf{h}_{\mathbf{c}}$ & GS & GS\% & $\mathbf{h}_{\mathbf{c}}$ & GS & $\mathbf{G S}_{\%}$ \\
\hline Planaltina(DF) & 0,95 & 332,0 & 22,9 & 0,95 & 403,5 & 27,8 \\
\hline Nova Porteirinha(MG) & 0,92 & 825,9 & 78,2 & 0,92 & 801,2 & 75,8 \\
\hline Pelotas(RS) & 0,79 & 930,0 & 180,0 & 0,79 & 913,7 & 176,8 \\
\hline \multicolumn{7}{|c|}{ Ganho estimado em relação à testemunha (CNPAE-154) } \\
\hline \multirow{2}{*}{ Ambiente } & \multicolumn{6}{|c|}{ Seleção baseada na médiaSeleção baseada no centroide } \\
\hline & $\mathbf{h}_{\mathbf{c}}$ & GS & $\mathbf{G S}_{\%}$ & $\mathbf{h}_{\mathbf{c}}$ & GS & $\mathbf{G S}_{\%}$ \\
\hline Planaltina(DF) & 0,95 & 0,0 & 0,0 & 0,95 & 41,7 & 2,3 \\
\hline Nova Porteirinha(MG) & 0,92 & 173,4 & 9,8 & 0,92 & 148,7 & 8,4 \\
\hline Pelotas(RS) & 0,79 & 608,8 & 65,9 & 0,79 & 592,5 & 64,1 \\
\hline
\end{tabular}

$\mathbf{h}_{\mathbf{c}}$ : acurácia do processo seletivo, GS: ganho de seleção em gramas.planta ${ }^{-1}, \mathbf{G S}_{\%}$ : ganho de seleção percentual. 
manso, a progênie CNPAE-154 tem sido cultivada em várias regiões e considerada como uma das mais produtivas. No entanto, observou-se que foram encontradas várias progênies que superaram o desempenho do CNPAE-154, que apresentou o $7^{\circ}$ melhor desempenho em Nova Porteirinha, o $8^{\circ}$ em Planaltina e o $15^{\circ}$ em Petrolina, onde esteve entre as progênies menos produtivas.

Ainda considerada em fase de domesticação, a viabilidade deste cultivo ainda depende de um incremento de produtividade, a ser realizado com a seleção de plantas e melhoria das condições de ambiente (Drumond et al., 2010; Gouveia et al., 2012; Laviola et al. 2012). As novas médias estimadas com o plantio das progênies selecionadas, em toneladas por hectare, foram de 2,34 ton.ha- ${ }^{-1}$, em Planaltina, de 2,37 ton.ha ${ }^{-1}$, em Nova Porteirinha, e de 2,09 ton.ha ${ }^{-1}$, em Pelotas - RS.

A estruturação das populações de melhoramento em progênies subsidia a seleção de características de baixa herdabilidade, considerando-se, em uma primeira etapa, a seleção entre progênies e, em uma segunda etapa, a seleção dentro de progênies (Resende, 2002, Cruz et al., 2004). Atualmente, a seleção dos melhores indivíduos dentro de progênies ainda depende do desenvolvimento de protocolos eficientes para propagação vegetativa do pinhãomanso, seja por estaquia ou por cultura de tecidos (Datta et al., 2007; Daudet et al., 2011). Os resultados deste estudo representam uma das primeiras caracterizações da interação genótipos $\mathrm{x}$ ambientes desta oleaginosa e servirão para seleção de materiais genéticos e para a tomada de decisão na condução de programa de melhoramento.

\section{CONCLUSÕES}

Foi verificada a ocorrência de interação genótipos $\mathrm{x}$ ambientes na expressão do caráter produção de grãos, em progênies de meios-irmãos de pinhão-manso, avaliadas em três regiões, no Brasil. Foram identificadas oito progênies de adaptabilidade geral, três progênies de baixa adaptabilidade, duas progênies de adaptabilidade específica a ambientes favoráveis, duas progênies de adaptabilidade específica a ambientes desfavoráveis em diferentes regiões do Brasil. As estimativas de progresso genético indicam a eficiência da seleção massal, realizada nos municípios de Petrolina, PE, e Nova Porteirinha, MG. Observa-se que os ganhos de seleção obtidos pelo método centroide são mais equilibrados entre ambientes e, por isso, preferíveis.

\section{AGRADECIMENTOS}

Os autores agradecem ao MCTI, à Finep e ao CNPq pelo apoio financeiro para a execução do projeto Pesquisa, Desenvolvimento e Inovação em Pinhão-Manso para Produção de Biodiesel (BRJATROPHA).

\section{REFERÊNCIAS}

Bahadur B, Sujatha M \& Carels N (2013) Jatropha, Challenges for a New Energy Crop: Genetic Improvement and Biotechnology. New York, Springer Verlag. 184p.

Borges CV, Ferreira FM, Rocha RB, Santos ARD \& Laviola BG (2014) Capacidade produtiva e progresso genético de pinhãomanso. Ciência Rural, 44:64-70.

Carels N, Sujatha M \& Bahadur B (2013) Jatropha, Challenges for a New Energy Crop: Farming, Economics and Biofuel. New York, Springer Verlag. 599p.

Cruz CD, Regazzi AJ \& Carneiro PCS (2004) Modelos Biométricos Aplicados ao Melhoramento Genético. Editora Viçosa, Viçosa. 480p.

Cruz CD \& Castoldi F (1991) Decomposição da interação genótipos $\mathrm{x}$ ambientes em partes simples e complexa. Revista Ceres, 38:422 430.

Cruz CD, Torres RA \& Vencovsky R (1989) An alternative approach to the stability analysis proposed by Silva and Barreto. Revista Brasileira de Genetica, 12:567-580.

Datta MM, Mukherjee P, Ghosh B \& Jha TB (2007) In vitro clonal propagation of biodiesel plant (Jatropha curcas L.). Current Science, 93:1438-1442.

Daudet MMS, Mergeai G, Baudoin JP \& Toussaint A (2011) In vitro culture of Jatropha curcas L. Biotechnologie Agronomie Societe Et Environnement, 15:567-574.

Dias LAS, Leme LP, Laviola BG, Pallini Filho A, Pereira OL, Carvalho M, Manfio CE, Santos AS, Sousa LCA, Oliveira TS \& Dias DCFS (2007) Cultivo de pinhão-manso (Jatropha curcas L.) para produção de óleo combustível. Viçosa, Universidade Federal de Viçosa. 40p.

Divakara BN, Upadhyaya HD, Wani SP \& Gowda CLL (2010) Biology and genetic improvement of Jatropha curcas L.: A review. Applied Energy, 87:732-742.

Drumond MA, Santos CAF, Oliveira VR, Martins JC, Anjos JB \& Evangelista MRV (2010) Agronomic performance of different genotypes of physic nut in the semi-arid zone of Pernambuco state. Ciência Rural, 40:44-47.

Eberhart SA \& Russel WA (1966) Stability parameters for comparing varieties. Crop Science, 6:36-40.

Falconer DS (1987) Introdução a genética quantitativa. Viçosa, Imprensa Universitária/UFV. 279p.

Filho RDCB, Pratissoli D, Nava DE, Monte FG, Guidoni AL, Silva SDDaE \& Polanczyk RA (2013) Development of Pachycoris torridus (Hemiptera: Scutelleridae) on Jatropha curcas (Euphorbiaceae), Psidium cattleianum (Myrtaceae) and Aleurites fordii (Euphorbiaceae). Florida Entomologist, 96:1149-1157.

Finlay KW \& Wilkinson GN (1963) The analysis of adaptation in a plant breeding programme. Australian Journal of Agricultural Research, 14:742-754.

Freitas RG, Missio RF, Matos FS, Resende MDV \& Dias LAS (2011) Genetic evaluation of Jatropha curcas: an important oilseed for biodiesel production. Genetics and Molecular Research, 10:14901498.

Gouveia EJ, Rocha RB, Laviola BG, Ramalho AR, Ferreira MDGR \& Dias LAS (2012) Aumento da produção de grãos de pinhãomanso pela aplicação de benziladenina. Pesquisa Agropecuaria Brasileira, 47:1541-1545.

Juhász ACP, Morais DLB, Soares BO, Pimenta S, Rabello HO \& Resende MDV (2010) Parâmetros genéticos e ganho com a seleção para populações de pinhão-manso (Jatropha curcas). Pesquisa Florestal Brasileira, 30:25-35. 
Juhász ACP, Resende MDV, Laviola BG \& Costa MR (2013) Melhoramento genético de Jatropha curcas: considerações e metodologias. In: Resende JCF de, Londe LN \& Neves W dos S (Eds.) Pinhão-manso. Nova Porteirinha, Epamig. p.89-154.

Laviola BG, Alves AA, Rocha RB \& Drumond MA (2012) The importance of Jatropha for Brazil. In: Carels N, Sujatha M \& Bahadur B (Eds.) Jatropha, Challenges for a New Energy Crop. New York, Springer Verlag. p.71-94.

Laviola BG \& Dias LAD (2008) Nutrient Concentration InJatropha curcas L. Leaves And Fruits And Estimated Extraction At Harvest. Revista Brasileira de Ciência do Solo, 32:1969-1975.

Lin CS (1982) Grouping genotypes by a cluster method directly related to genotype environment interaction mean square. Theoretical and Applied Genetics, 62:277-280.

Lin CS and Binns MR (1988) A superiority measure of cultivar performance for cultivar $\mathrm{x}$ location data. Canadian Journal of Plant Science, 68:193-198.

Mishra DK (2009) Selection of candidate plus phenotypes of Jatropha curcas L. using method of paired comparisons. Biomass and Bioenergy, 33:542-45.

Nascimento M, Cruz CD, Campana ACM, Tomaz RS, Salgado CC \& Ferreira RDP (2009) Alteração no método centroide de avaliação da adaptabilidade genotípica. Pesquisa Agropecuária Brasileira, 44:263-269.

Plaisted RL \& Peterson LC (1959) A technique for evaluating the ability of selections to yield consistently in different locations or seasons. American Potato Journal, 36:381-385.

Resende JCF, Londe LN \& Neves WS (2013) Pinhão-manso. Nova Porteirinha, Epamig. 524p.
Resende MDV (2002) Genética Biométrica e Estatística no Melhoramento de Plantas Perenes. Brasília, Embrapa Informação Tecnológica. $975 \mathrm{p}$.

Resende MDV (2007) Matemática e estatística na análise de experimentos e no melhoramento genético. Colombo, Embrapa Florestas. $561 \mathrm{p}$.

Resende MDV, Furlani-Júnior E, Moraes MLT \& Fazuoli LC (2001) Estimativas de parâmetros genéticos e predição de valores genotípicos no melhoramento do cafeeiro pelo procedimento REML/BLUP. Bragantia, 60:185-193.

Rocha RB, Muro-Abad JI, Araújo EF \& Cruz CD (2005) Utilização do método centróide para estudo da estabilidade e adaptabilidade ao ambiente. Ciência Florestal, 15:255-266.

Rosado TB, Laviola BG, Faria DA, Pappas MR, Bhering LL, Quirino B, Grattapaglia D (2010) Molecular markers reveal limited genetic diversity in a large germplasm collection of the biofuel crop Jatropha curcas L. in Brazil. Crop Science. 50: 2372-82.

Spinelli VM, Rocha RB, Ramalho AR, Marcolan AL, Vieira Junior JR, Fernandes CDF, Linhares Teixeira Militao JS \& Dos Santos Dias LA (2010) Primary and secondary yield components of the oil in physic nut (Jatropha curcas L.). Ciência Rural, 40:1752-1758.

Tominaga N, Kakida J \& Yasuda EK (2007) Cultivo de pinhãomanso para produção de biodiesel. Viçosa, CPT. 220p.

Verma MM, Chahal GS \& Murty BR (1978) Limitations of conventional regression analysis: a proposed modification. Theoretical and Applied Genetics, 53:89-91. 